\title{
The Longitudinal Temperature Detection System and Its Application of the Distributed Optical Fiber Freezer
}

\author{
Zheng Xiaoliang ${ }^{1,2, *}$, Sheng Huajun ${ }^{3}$ and Hu Yelin ${ }^{2}$ \\ ${ }^{I}$ School of Mining and Safety Engineering, Anhui University of Science and Technology, Huainan, Anhui, P.R. China \\ ${ }^{2}$ School of Electric and Information Engineering, Anhui University of Science and Technology, Huainan, Anhui, P.R. \\ China \\ ${ }^{3}$ China Coal Construction (Group) Corporation Ltd., Hefei, Anhui, P.R. China
}

\begin{abstract}
The modeling illustrates the equation of the relation between the temperature of the freezer and the radius of the tjaele, so the horizon tjaele radius can be calculated by the temperature of the horizon freezer. Compared with the single bus temperature sensor system, the former can measure the continuous longitudinal temperature distribution at the spatial resolution of $1 \mathrm{~m}$. Thus the relevant tjaele radius can be calculated, and the same is for the whole longitudinal direction. Therefore, the closure plans of ice wall can be examined by the freezer overlay. The study firstly gives us an introduction of the construction, working principle and methods of the distributed optical fiber freezer, and then shows some examples.
\end{abstract}

Keywords: Freezing method, Frozen wall, Longitudinal temperature, Temperature field, Distributed temperature sensing.

\section{INTRODUCTION}

The freezing method is a special construction method in efficiently penetrating unstable stratum like deep alluvium and soft rock, which can be seen in the coal mine shaft construction. More specifically, before digging, the surrounding rock is firstly frozen into closed frozen wall through artificial refrigeration in order to resist the pressure from water and soil and cut the contact between the underground water and the shaft. Secondly, the construction is started under the protection of the frozen wall, and now the freezing depth of the shaft has already surpassed seven hundred meters [1, 2]. Normally, there are 4 to 7 thermometer holes distributed in the inside and outside the arranged circle of the freezer, so the situation of the frozen wall can be observed. However actually, because of the complicated geological conditions, it's quite difficult to fully know the state of the frozen wall just through the data of these thermometer holes. Especially under conditions like some special freezing constructions or the circles don't cross each other on the frozen wall, which makes it really hard to know the state and detect the concrete location of these circles [3]. The advanced distributed temperature sensing can better reflect the temperature conduction radius of the frozen soil columns and the radius of the frozen soil itself so that we can grasp the condition of the frozen wall and find the malfunction location and then promote the freezing construction.

*Address correspondence to this author at the School of Electric and Information Engineering, Anhui University of Science and Technology, Huainan, Anhui, 232001, P.R. China; Tel: +86 05546668624 ;

Fax:+860554 6668624; E-mail: zhengx1@aust.edu.cn

\section{THE LONGITUDINAL TEMPERATURE DETEC- TION SYSTEM DISTRIBUTED OPTICAL FIBER FREEZER}

The commonly used temperature detection sensor is single bus digital temperature sensor DS18B20. When several DS18B20s are concatenated together on a cable with a certain distance and then by putting the cable into the thermometer hole or the freezer, we can get different temperature value. Whereas, there exist many weaknesses, such as limited sensor number, long and discontinuous temperature measuring distance. Moreover, considering there is only one cable, so the fault rate is quite high; and the drive capability of the sensor is also limited because of the need of power supply.

\subsection{System Components}

The distributed optical fiber sensing technology possesses a great sense system, a high level of spatial resolution, a continuous detection spot and a long measure distance because it is based on the scattering during the transmission of laser pulse. The system consists of a distributed optical fiber temperature interrogator, an optical fiber detection retractable frame, a freezer guide pulley and a temperature measuring optical fiber cable, as shown in Fig. (1). Specifically, the cable is used for detecting the longitudinal temperature of the freezer, meanwhile, the pulley and frame aim at receiving and relieving the detected fiber cable. Finally, the interrogator is used for observing and controlling the signal.

The structure of distributed optical fiber temperature interrogator is as shown in Fig. (2), mainly consisting of optics part, signal collection and processing part. The former is 


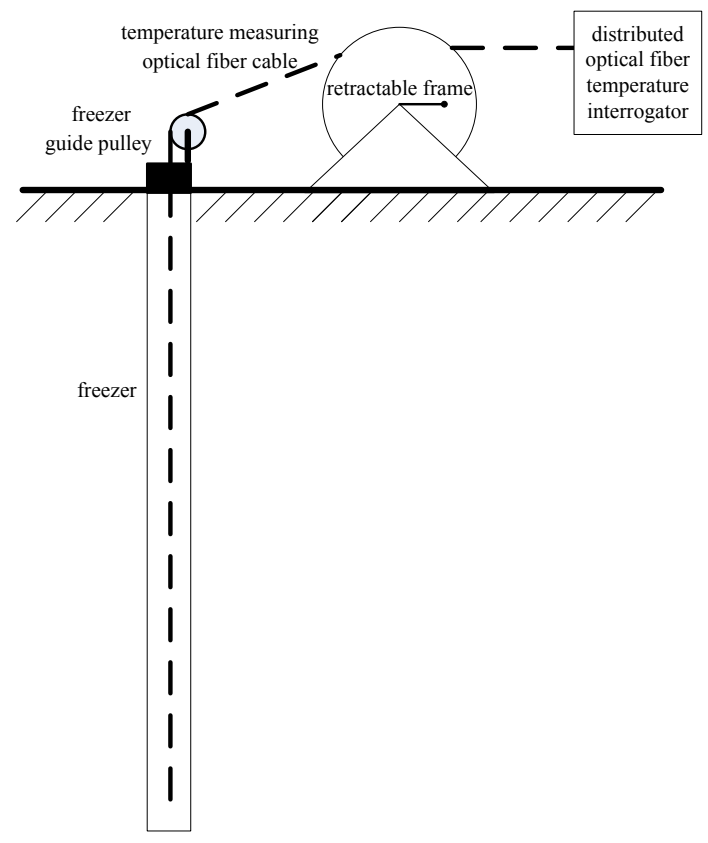

Fig. (1). Components of the detection system.

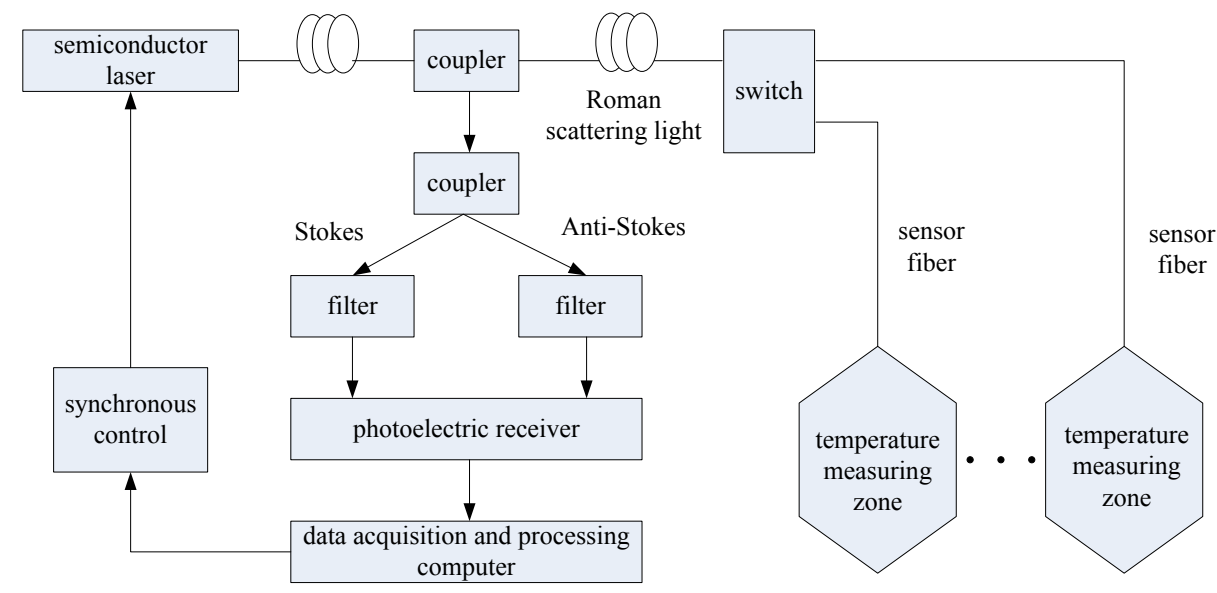

Fig. (2). Structure of the distributed optical fiber temperature interrogator.

further composed of the semiconductor laser, the optical fiber directional coupler, the sensor fiber and the optical spectral components, while the latter consists of the photoelectric receiver, and the data acquisition and processing computer [4-7].

\subsection{The Working Principle of the Distributed Optical Fiber Temperature Interrogator}

The system is able to observe the longitudinal temperature change of the whole temperature field with a certain interval. The measure principle is OTDR (Optical Time Domain Reflectometry) and Raman Scattering Temperature Effect, which can not only locate the temperature measuring point but also perceive and measure the temperature.

In the perspective of energy in quantum theory, Raman Scattering is caused by the inelastic collision of photon. It is found that the Anti-Stokes scattering light is quite sensitive to temperature change; while the Stokes scattering light also has something to do with the temperature to a smaller extent; whereas, Rayleigh scattering light has nothing to do with the temperature.

The Anti-Stokes scattering photon number at optical fiber zone $\mathrm{L}$ is:

$N_{a}=K_{a} S v_{a}^{4} N_{e} \exp \left[-\left(a_{0}+a_{a}\right) L\right] R_{a}(T)$

The Stokes scattering photon number at optical fiber zone $\mathrm{L}$ is:

$N_{s}=K_{s} S v_{s}^{4} N_{e} \exp \left[-\left(a_{0}+a_{s}\right) L\right] R_{s}(T)$

The Rayleigh scattering photon number at optical fiber zone $\mathrm{L}$ is: 


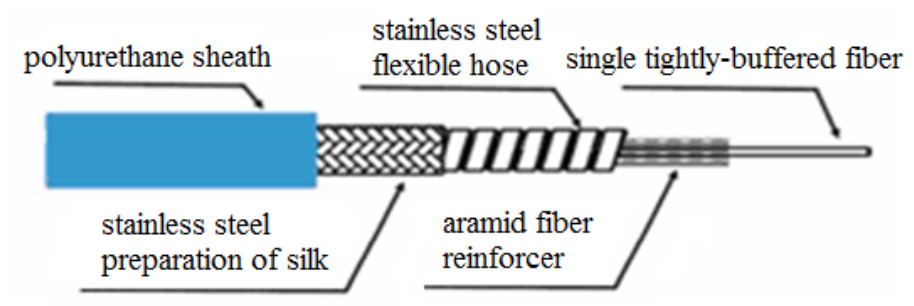

Fig. (3). The armored cable structure.

$N_{R}=K_{R} S v_{0}^{4} N_{e} \exp \left(-2 a_{0} L\right)$

$\mathrm{Ne}$ represents the optical fiber incident light pulse photon number; $K R, K a, K s$ respectively mean the related coefficients of the scattering cross sections of Rayleigh, AntiStokes and Stokes; $S$ is the backward scattering factor of the optical fiber; $v_{0}, v_{a}, v_{s}$ are the scattering light frequency of the three; and $a_{0}, a_{a}, a_{s}$ mean the transmission loss of them; meanwhile $L$ is the distance between the area to be measured and the irradiation port. $R_{a}(T), R_{s}(T)$ are the related coefficients, and it is related to the temperature in the optical fiber area:

$$
\begin{aligned}
& R_{a}(T)=[\exp (h \Delta v / k T)-1]^{-1} \\
& R_{s}(T)=[1-\exp (-h \Delta v / k T)]^{-1}
\end{aligned}
$$

$\Delta v$ is the frequency of Raman scattering light; $h$ is Planck constant; and $k$ is Boltzmann coefficient.

The principle of temperature demodulation method is demodulating Anti-Stokes scattering curve OTDR with Stokes scattering curve OTDR, so from (1) and (2), we can get (6):

$\frac{N_{a}(T) N_{s}\left(T_{0}\right)}{N_{s}(T) N_{a}\left(T_{0}\right)}=\frac{\exp (-h \Delta v / k T)}{\exp \left(-h \Delta v / k T_{0}\right)}$

From (6), we know:

$\frac{1}{T}=\frac{1}{T_{0}}-\frac{k}{h \Delta v} \ln \frac{N_{a}(T) N_{s}\left(T_{0}\right)}{N_{s}(T) N_{a}\left(T_{0}\right)}$

$T_{0}, N_{a}\left(T_{0}\right), N_{s}\left(T_{0}\right), N_{a}(T)$ and $N_{s}(T)$ are all known, so we can get the temperature $T$.

\subsection{The Longitudinal Temperature Measuring Method and the Sensor Fiber Structure}

The longitudinal temperature measuring is action-based measuring. Firstly, stop the brine circulation, secondly, put the temperature measuring optical cable into the freezer, and then after the longitudinal detection, take out the cable and finally recover the brine circulation. And the whole process probably continues about one hour. As a result, based on the analysis, during the initial stage, temperature is quite sensitive to the size of the frozen soil column, so we should complete the detection of one freezer within four hour. Of course, we can allocate time accordingly under different circumstances to set up each schedule and finish all the detection well.
Considering the reiterative pulling down and back, the cable needs to be quite tensile in order to avoid any mistake, and the structure is as shown in Fig. (3). We should put both the single tightly-buffered fiber and the aramid fiber together into the stainless steel flexible hose, and meanwhile add a layer of stainless steel preparation of silk onto the hose to increase the tensile ability. Finally put a polyurethane sheath to resist low temperature. Besides, in order to prevent the cable from fracture during the process, we should use the colligation method together with a $1.8 \mathrm{~mm}$ galvanized steel wire rope as the tension carrier. The concrete parameter is as follows: the diameter of multimode fiber core is $62.5 \pm 2.5 \mu \mathrm{m}$ with a maximum attenuation speed of $3.5 \mathrm{~dB} / \mathrm{km}(850 \mathrm{~nm})$ and $1.5 \mathrm{~dB} / \mathrm{km}(1300 \mathrm{~nm})$, and the working temperature is from -50 degree to 90 degree, with a dynamic bending radius of $10 \mathrm{D}$ and static bending radius of $20 \mathrm{D}$, together with a long-term tensile strength of $200 \mathrm{~N}$ and a short-term tensile strength of $300 \mathrm{~N}$. Moreover, the longtime compressive reinforcement is $3000 \mathrm{~N} / 100 \mathrm{~mm}$ and short time is $5000 \mathrm{~N} / 100 \mathrm{~mm}$ with a weight of $25 \mathrm{Kg} / \mathrm{Km}$.

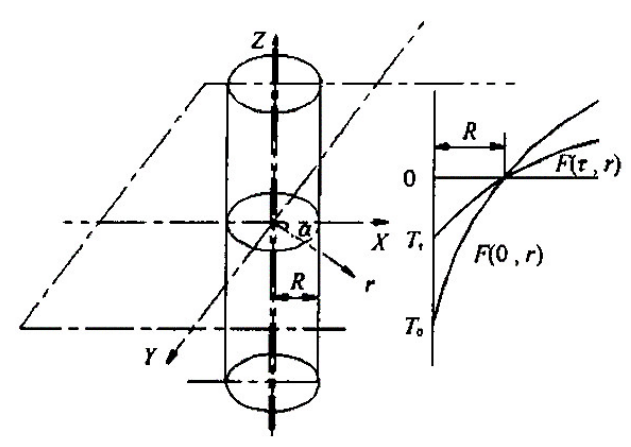

Fig. (4). The mathematical model of temperature rise in single span frozen column.

\section{THE MATHEMATICAL MODEL OF TEMPER- ATUE RISE IN THE TEMPERATURE FIELD INSIDE THE FROZEN SOIL COLUMN [3, 8-10]}

The longitudinal temperature detection is to stop the brine circulation in a planned way during the freezing period and then put down the optical cable to measure the longitudinal temperature, and according to the temperature rise after freezing, we can analyze the detailed situation of the frozen wall.

If we regard the length of freezer as infinite to build the simplified model of the frozen soil column as Fig. (4), after the heat exchange for some time, the frozen soil keeps ex- 
panding to form a frozen column with. And if at a moment $t$, we stop the brine circulation, during this period, there is a plane transient heat conduction, so the column has an instantaneous temperature rise. Based on the flat heat conduction principle of the diameter of the polar coordinates to the axial symmetry, we could get the differential equation as below:

$\frac{\partial T}{\partial \tau}=\alpha\left(\frac{\partial^{2} T}{\partial r^{2}}+\frac{1}{r} \frac{\partial T}{\partial r}\right)$

$T$ is the temperature; $\mathrm{t}$ is time; $\mathrm{r}$ is radius.

If the thermal diffusivity were a parameter to reflect the heat-conducting property, the boundary condition would set up the interface temperature of the frozen soil and melted soil as 0 degree and then stop the freezing process for a while with the frozen soil radius unchanged. Because the heat exchange between the frozen soil and melted soil mainly causes the temperature rise within the column, the frozen soil won't melt. Use $T(\tau, R)=0\left(0 \leq \tau \leq \tau_{0}\right)$, where $\tau_{0}$ is the longest unfrozen time.

The initial condition is the initial temperature field function before the temperature rise.

$$
\begin{aligned}
& F(r)=\sum_{n=1}^{N}(-1)^{n-1}\left(\frac{r}{2}-1\right)^{n} / n(0<r \leq 4) \\
& T(0, r)=A F(r)+B\left(r_{0}<r \leq R\right)
\end{aligned}
$$

$A$ and $B$ are the coefficients of the function, while $r 0$ is the outside radius of the freezing pipe.

The conditions of the initial temperature field are: $\left.T_{0}\right|_{r=R}=0,\left.T\right|_{r=r_{0}}=T_{0}$, normally $T_{0}$ is between -30 degree and -20 degree.

According to the principle of special function and variables separation method, we can deduce the temperature distribution inside the column at time $\mathrm{t}$, and as for different frozen column $\mathrm{R}$, the temperature distribution will be as:

$$
\begin{aligned}
& T(r, R, \tau)=\sum_{n=1}^{\infty} C_{n} e^{-\alpha\left[\frac{q_{n}^{(0)}}{R}\right]^{2} \tau} J_{0}\left(\frac{q_{n}^{(0)}}{R} r\right) \\
& C_{n}=\frac{2}{\left[J_{0}\left(q_{n}^{(0)}\right)\right]^{2} R^{2}} \int_{0}^{R} r F(r) J_{0}\left(\frac{q_{n}^{(0)}}{R} r\right) d r
\end{aligned}
$$

A is the thermal diffusivity of the frozen soil; $J_{0}$ is the zero order Bessel function; $q_{n}^{(0)}$ is the positive null point of $J_{0}$. $n$ could be $1,2,3$ and so on.

The equation (11) is a series consisting of coefficient, exponential function and Bessel function compound, and

because $r_{0}$ is quite small, while $\mathrm{r}$ approximates 0 , the temperature will be quite similar to $T_{r_{0}}$ of the measuring point in the freezer.
$\left.T_{r_{0}} \approx T(r, R, \tau)\right|_{r \rightarrow 0}=\sum_{n=1}^{\infty} C_{n} e^{-\alpha\left[\frac{q_{n}^{(0)}}{R}\right]^{2} r}$

Via derivate $t$ in (13), we can get the temperature rise speed in the freezer as

$\left.\dot{T}_{r_{0}}\right|_{\tau}=-\frac{\alpha}{R^{2}} \sum_{n=1}^{\infty} C_{n}\left[q_{n}^{(0)}\right]^{2} e^{-\alpha\left[\frac{q_{n}^{(0)}}{R}\right]^{2}} r$

When $\mathrm{t}=0$, according to (13) and (14), we can get

$$
\begin{aligned}
& T_{r_{0}} \approx \sum_{n=1}^{\infty} C_{n} \\
& \left.\dot{T_{r_{0}}}\right|_{\tau}=-\frac{\alpha}{R^{2}} \sum_{n=1}^{\infty} C_{n}\left[q_{n}^{(0)}\right]^{2}
\end{aligned}
$$

As a result, $\mathrm{Cn}$ depends on the initial temperature field before the unfrozen period. However, the convergence value of $\sum_{n=1}^{\infty} C_{n}$ approximately equals to the initial temperature $T_{0}$.

According to the above equation, we know the longitudinal temperature can be used to define the radius of frozen soil surrounding the freezer. And based on the unfrozen time, we can detect the longitudinal temperature, and then use equation (13) to know the exclusive temperature of each point at different radius and moments. What's more, the radius of frozen soil can also be measured. Thus we can use figure to show the relation between the size and scale of the frozen soil radius, and then add all the radius surrounding the freezer at the same layer together, so we can directly see the situation of the frozen wall.

\section{APPLICATION EXAMPLES}

There happened a water and sand crush accident in the auxiliary shaft, causing the shaft destruction in a coal mine in Huainan, Anhui province. So it was decided to use the ground pregrouting and freezing method to restore the accident. The auxiliary shaft was equipped with double row holes with 42 freezing holes outside and 32 inside. After 75 days' working, the water level of 4 hydrological holes all reached to the pipe orifice. The shaft was already built, so it was difficult to decide about the real situation of the frozen wall either by the water from hydrological hole or by the temperature of the measuring hole. In the end, in order to know the real condition of the frozen wall, after 131 days' freezing, they decided to use the longitudinal temperature detection system of distributed optical fiber freezer.

During the detection, they used two sets of the systems at the same time with 75 time schedule of frozen and unfrozen period in three days. Therefore, for each freezer the longitudinal temperature value could be obtained from ground to 


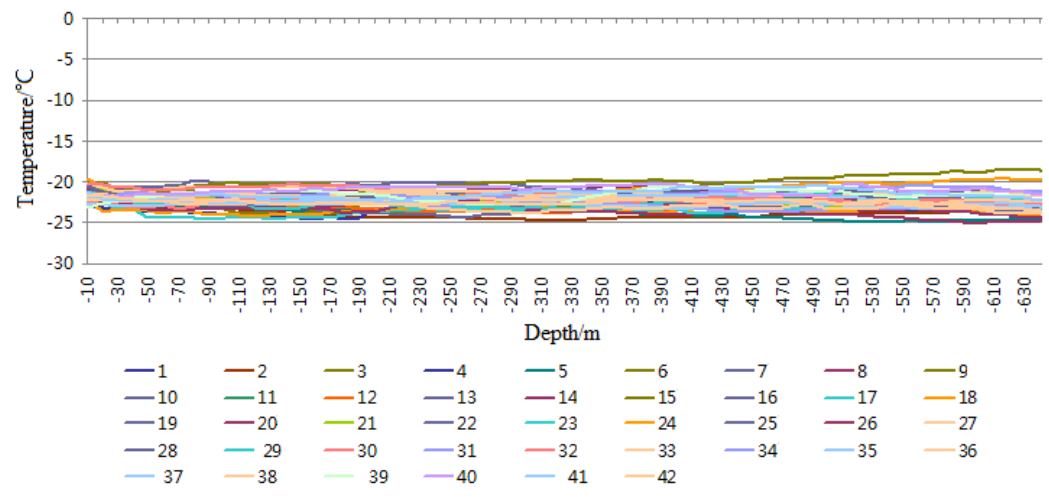

Fig. (5). The longitudinal temperature curve of outside freezer in the auxiliary shaft.

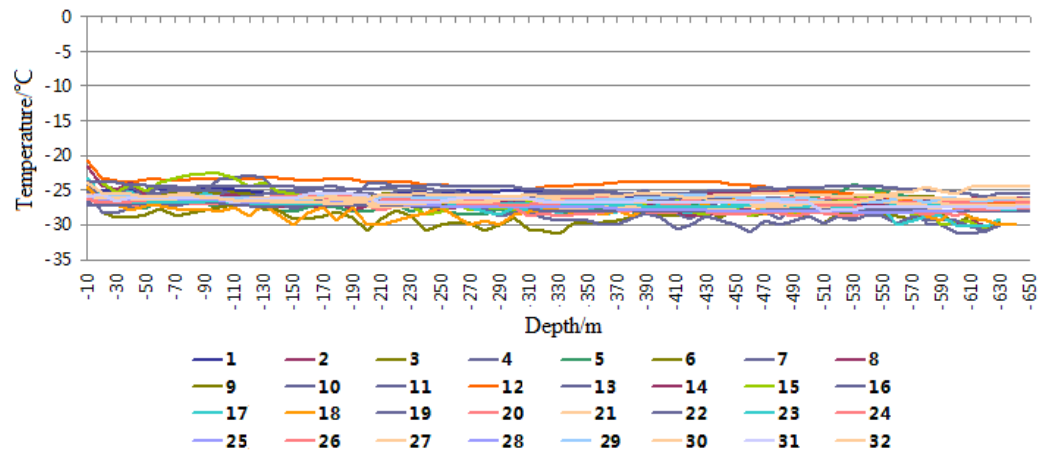

Fig. (6). The longitudinal temperature curve of inside freezer in the auxiliary shaft.

underground with a spatial resolution of $1 \mathrm{~m}$, and they got the longitudinal temperature curve for all the freezer as shown in Figs. (5) and (6). It was concluded that the whole temperature change was from -30 degree to -19 degree, so the freezing hole of the auxiliary shaft was in good condition without any abnormal phenomena. Besides, the deeper the shaft, the lower the longitudinal temperature, and the temperature inside freeze was lower than that of outside, which conformed to the direct circulation of the frozen brine.

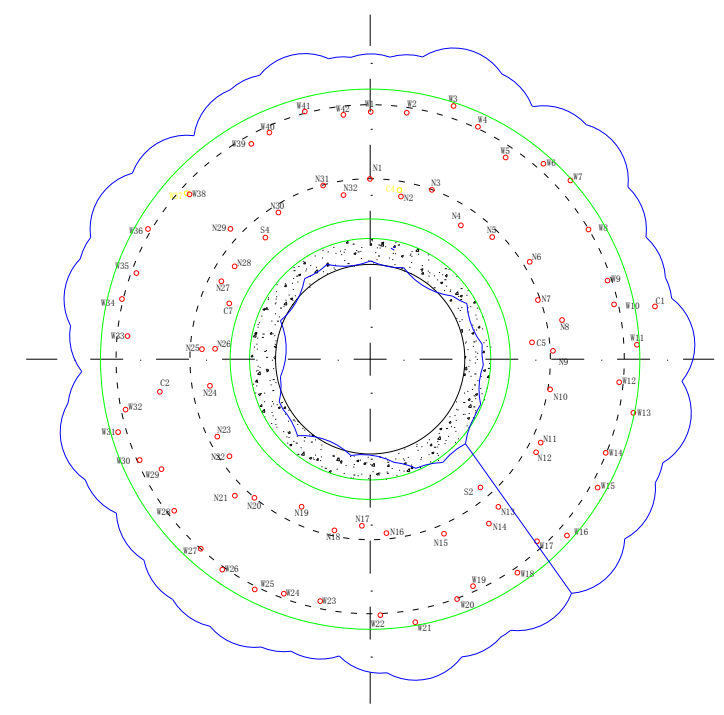

Fig. (7). The circular diagram of the frozen wall at a depth of $490 \mathrm{~m}$.

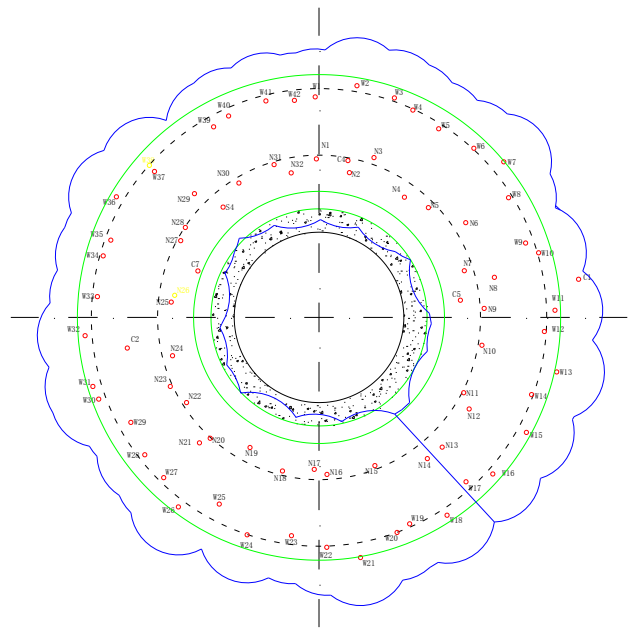

Fig. (8). The circular diagram of the frozen wall at a depth of $560 \mathrm{~m}$.

According to equation (13), we can get the frozen soil radius of each freezer. If we add the radius of a certain layer, we will know the condition of the whole frozen wall of this layer. Figs. (7) and (8) respectively gives us two circular diagrams of the frozen wall at depths of $-490 \mathrm{~m}$ and $-560 \mathrm{~m}$, from which we can see no abnormity. The system detects each temperature value at every single meter, so we can get a circular diagram for every single meter. And then by adding all the diagrams in longitudinal directions, we can draw a solid figure to show the condition of frozen wall more directly and precisely. 


\section{CONCLUSION}

Compared with the traditional single bus temperature sensor system, the distributed system is much more concrete and accurate with no blind point in detection, so we can get the continuous temperature curve to directly show the longitudinal temperature change in the freezer. According to the relation between the temperature change of the freezer and the radius of frozen soil, we can know each outside column radius of the freezer from the longitudinal temperature. Thus, we can get the circular diagram of a certain layer to reflect the development condition of the frozen wall, which is much more accurate compared with the data detected from several separate temperature measuring holes. It is confirmed that the system features a better characteristic so that it serves as a new detection method to help people better control the freezing construction. Therefore, the whole construction process can be much safer and faster.

\section{CONFLICT OF INTEREST}

The authors confirm that this article content has no conflict of interest.

\section{ACKNOWLEDGEMENTS}

Declared none.

\section{REFERENCES}

[1] G. Yang, and J. Xi, "Review and prospects of research on freezing design theory of coal mine shaft", Chinese Journal of Underground Space and Engineering, vol. 6, no. 3, pp. 627-635, 2010.
[2] L. Song, W. Yang, and H. Li, "Monitoring of freezing shaft sinking in Ultra-deep alluvium of Guotun coal mine", Journal of Mining \& Safety Engineering, vol. 27, no. 1, pp. 19-23, 2010.

[3] X. Zhou, and X. Zhang, "Research on the thickness estimation of freezing wall according to temperature measurement along freeze tube", Journal of China Coal Society, vol. 28, no. 2, pp. 162-166, 2002.

[4] Z. Guo, X. Zheng, Z. Lu, and 1. Ma, "Technology and application of distributed optical fiber temperature sensor", Journal of CAEIT, vol. 3 , no. 5, pp. 543-546, 2008.

[5] J. Wang, Z. Zhang, H. Xu, H. Liu, X. Yu, and I. S. Kim, "Research of a new temperature measure principle of distributed optical fiber temperature sensors", Journal of China Jiliang University, vol. 17, no. 1, pp. 25-28, 2006

[6] X. Zheng, Z. Guo, H. Xie, and L. Ma, "Monitoring and measurement system of freezing temperature field based on distributed optical fiber sensor technology", Coal Science and Technology, vol. 37, no. 1, pp. 18-21, 2009.

[7] N. Ding, C. Hu, J. Yu, and Y. Liu, "Application of distributed fiber-optic temperature sensor in shaft-sinking by freezing in coal mine", Transducer and Microsystem Technologies, vol. 33, no. 6, pp. 154-156, 2014.

[8] X. Hu, F. Huang, and N. Bai, "Models of artificial frozen temperature field considering soil freezing point", Journal of China University of Mining \& Technology (Natural Science), vol. 37, no. 4, pp. 550-555, 2008 .

[9] X. Hu, S. She, and R. Yu, "Average temperature calculation for straight single-row-piped frozen soil wall", Sciences in Cold and Arid Regions, vol. 3, no. 2, pp. 124-131, 2011.

[10] X. Hu, J. Chen, Y. Wang, and W. Li, "Analytical solution to steady-state temperature field of single-circle-pipe freezing", Rock and Soil Mechanics, vol. 34, no. 3, pp. 874-880, 2013. 